zu dient, den »Souverän « der BRD auf einen Notstand vorzubereiten, dessen Beseitigung ohne Stärkung des demokratischen und sozialistischen Potentials nur in faschistischer Weise vorstellbar ist. ${ }^{57}$

Gerd Temming

\title{
Karl Marx über die spositiven< Seiten des Verbrechens - oder die Folgen einer Glosse in der westlichen Kriminalsoziologie
}

\section{I.}

\author{
»Abschweifung 1 \\ (über produktive Arbeit)
}

Ein Philosoph produziert Ideen, ein Poet Gedichte, ein Pastor Predigten, ein Professor Kompendien usw. Ein Verbrecher produziert Verbrechen. Betrachtet man näher den Zusammenhang dieses letzten Produktionszweigs mir dem Ganzen der Gesellschaft, so wird man von vielen Vorurteilen zurückkommen. Der Verbrecher produziert nicht nur Verbrechen, sondern auch das Kriminalrecht und damit auch den Professor, der Vorlesungen über das Kriminalrecht hält, und zudem das unvermeidliche Kompendium, worin dieser selbe Professor seine Vorträge als »Ware« auf den allgemeinen Markt wirft. Damit tritt Vermehrung des Nationalreichtums ein. Ganz abgesehen von dem Privatgenuß, den, wie uns ein kompetenter Zeuge, Prof. Roscher, [sagt,] das Manuskript des Kompendiums seinem Urheber selbst gewährt.

Der Verbrecher produziert ferner die ganze Polizei und Kriminaljustiz, Schergen, Richter, Henker, Geschworene usw.; und alle seine verschiedenen Gewerbszweige, die ebenso viele Kategorien der gesellschaftlichen Teilung der Arbeit bilden, entwickeln verschiedene Fähigkeiten des menschlichen Geistes, schaffen neue Bedürfnisse und neue Weisen ihrer Befriedigung. Die Tortur allein hat zu den sinnreichsten mechanischen Erfindungen Anlaß gegeben und in der Produktion ihrer Werkzeuge eine Masse ehrsamer Handwerksleute beschäftigt.

Der Verbrecher produziert einen Eindruck, teils moralisch, teils tragisch, je nachdem, und leistet so der Bewegung der moralischen und ästhetischen Gefühle des Publikums einen "Dienst«. Er produziert nicht nur Kompendien über das Kriminalrecht, nicht nur Strafgesetzbücher und damit Strafgesetzgeber, sondern auch Kunst, schöne Literatur, Romane und sogar Tragödien, wie nicht nur Müllners »Schuld « und Schillers "Räuber«, sondern selbst »Odipus « und »Richard der Dritte« beweisen. Der Verbrecher unterbricht die Monotonie und

57 Der sozialpsychologisch notwendige Autbau eines inneren Feindes ist bereits in vollem Gange, vg1. Brüdkner/Krovoza, a. a. O. (FN 2), S. 6r ff. Das rechtliche Instrumentarium ist dank der Notstandsgesetzgebung vorhanden. Zur Vorbereitung eignet sich vorzüglich der Einsatz des Bundesgrenzschutzes aach Art 35 Abs. 2 GG.

$1 \mathrm{MEW}, \mathrm{Bd}$. 26.I, S. $363 \mathrm{~F}$. Der in Abschnite I folgende Text ist ein ironischer Exkurs im Kontext einer ausführlichen Auseinandersetzung mit vulgärökonomischen Auffassungen über die Produktivität aller Berufe in den *Theorien über den Mehrwert*. 
Alltagssicherheit des bürgerlichen Lebens. Er bewahrt es damit vor Stagnation und ruft jene unruhige Spannung und Beweglichkeit hervor, ohne die selbst der Stachel der Konkurrenz abstumpfen würde. Er gibt so den produktiven Kräften einen Sporn. Während das Verbrechen einen Teil der überzähligen Bevölkerung dem Arbeitsmarkt entzieht und damit die Konkurrenz unter den Arbeitern vermindert, zu einem gewissen Punkt den Fall des Arbeitslohns unter das Minimum verhindert, absorbiert der Kampf gegen das Verbrechen einen anderen Teil derselben Bevölkerung. Der Verbrecher tritt so als eine jener natürlichen "Ausgleichungen « ein, die ein richtiges Niveau herstellen und eine ganze Perspek tive »nützlicher « Beschäftigungszweige auftun.

Bis ins Detail können die Einwirkungen des Verbrechers auf die Entwicklung der Produktivkraft nachgewiesen werden. Wären Schlösser je zu ihrer jetzigen Vollkommenheit gediehn, wenn es keine Diebe gäbe? Wäre die Fabrikation von Banknoten zu ihrer gegenwärtigen Vollendung gediehn, gäbe es keine Falschmünzer? Hätte das Mikroskop seinen Weg in die gewöhnliche kommerzielle Sphäre gefunden (siehe Babbage) ohne Betrug im Handel? Verdankt die praktische Chemie nicht ebensoviel der Warenverfälschung und dem Bestreben, sie aufzudecken, als dem ehrlichen Produktionseifer? Das Verbrechen, durch die stets neuen Mittel des Angriffs auf das Eigentum, ruft stets neue Verteidigungsmittel ins Leben und wirkt damit ganz so produktiv wie strikes auf Erfindung von Maschinen. Und verläßt man die Sphäre des Privatverbrechens: Ohne nationale Verbrechen, wäre je der Weltmarkt entstanden? Ja, auch nur Nationen? Und ist der Baum der Sünde nicht zugleich der Baum der Erkenntnis seit Adams Zeiten her? Mandeville in seiner "Fable of the Bees" (1705) hatte schon die Produktivität aller möglichen Berufsweisen usw. bewiesen und überhaupt die Tendenz dieses ganzen Arguments:

*Das, was wir in dieser Welt das Böse nennen, das moralisch so gut wie das natürliche ist, ist das große Prinzip, das uns zu sozialen Geschöpfen macht, die feste Basis, das Leben und die Stütze aller Gewerbe und Bescbäftigungen ohne Ausnahme; hier haben wir den wahren Ursprung aller Künste und $\mathbb{W}$ issenschaften zu suchen; und in dem Moment, da das Böse aufhörte, müßte die Gesellschaft verderben, wenn nicht gar gänzlich untergehen.«

Nur war Mandeville natürlich unendlich kühner und ehrlicher als die philisterhaften Apologeten der bürgerlichen Gesellschaft.

II.

Nicht mehr aufzuhalten ist die Marx-Rezeption in der bürgerlichen Kriminalsoziologie.

1965: In England erscheint ein Sammelband, der neben anderen Texten auch Marx' Glosse "Abschweifung über produktive Arbeit« enthält. In einem "Brevier des schwarzen Humors" hätte dieses kleine satirische Meisterwerk gewiß seinen richtigen Platz. Wie der Titel dieses Readers jedoch zeigt, hatten die Herausgeber andere Auswahlkriterien: »Karl Marx - Ausgewählte Schriften zur Soziologie und Sozialphilosophie «.2 Dieses Mißverständnis, das mehr über das Niveau bürgerlicher Soziologie aussagt, als deren Vertretern lieb sein könnte,

2 Bottomore, T. B. und M. Rubel (Hrsg.), »Karl Marx: Selected Writings in Sociology and Social Philosophy «, London 1956. 
beeinflußt in den folgenden Jahren ungemein fruchtbar die Kriminalsoziologie.

1962: Lewis A. Coser publiziert im »American Journal of Sociology" seinen Aufsatz "Einige Funktionen abweichenden Verhaltens und normativer Flexibilität « ${ }^{3}$ und weist, sich u. a. auf diesen Marx-Text beziehend, mit wissenschaftlicher Akribie alle absurden Positionen zurück, die im Verbrechen nur eine dysfunktionale, negative Erscheinung sehen. Bereits Marx, Durkheim und Mead hätten erkannt, daß Gesellschaft zur Dynamisierung, als Motor gesellschaftlicher Entwicklung, das Verbrechen notwendig brauche. Heißt es nicht bereits bei Marx: „Er (der Verbrecher) bewahrt es (das bürgerliche Leben) damit vor Stagnation und ruft jene unruhige Spannung und Beweglichkeit hervor, ohne die selbst der Stachel der Konkurrenz abstumpfen würde. Er gibt so den produktiven Kräften einen Sporn «. Weiterhin, bereits Marx hätte argumentierend erkannt, daß Verbrechen für die gesellschaftliche Kohäsion einen unaufgebbaren Beitrag leiste (ähnlich wie Mead ${ }^{4}$ ): "Der Verbrecher produziert einen Eindruck, teils moralisch, teils tragisch, je nachdem, und leistet so der Bewegung der moralischen und ästhetischen Gefühle des Publikums einen `Dienst« «.

Die positiven volkswirtschaftlichen Auswirkungen des Verbrechens, von Marx so detailliert beschrieben, hatten bereits 1936 Hawkins und Waller in ihren »Kritischen Anmerkungen über die Kosten des Verbrechens« unabhängig von Marx entdeckt. ${ }^{5}$

Dieses strukturell-funktionale Denken von Marx, die Anerkennung prästabilisierter sozialer Systeme durch systemimmanente Gleichgewichtsprozesse, kommt, darauf verweist E. M. Schur ${ }^{6}$, besonders in folgender Formulierung zum Ausdruck: "Der Verbrecher tritt so als eine jener natürlichen >Ausgleichungene ein, die ein richtiges Niveau herstellen und eine ganze Perspektive snützlicher Beschäftigungszweige aufrun. «

Nachdem amerikanische Soziologen Marx wissenschaftlich rehabilitiert haben, beginnt die Rezeption auch in der westdeutschen Kriminalsoziologie.

I 968 nehmen F. Sads und R. König in die Bibliographie ihres Readers "Kriminalsoziologie « ${ }^{7}$ bereits die gesamte MEW-Ausgabe, »plus Ergänzungsband $\kappa$, auf. Noch im selben Jahr entdedst auch Günther Kaiser den betreffenden Abschnitt aus den »Theorien über den Mehrwert « und widerlegt mit Marx den genauso absurden wie mit einer marxistischen Position nicht vereinbaren Anspruch sozialistischer Kriminologen »die Kriminalität zu ,verändern', d. h. auszumerzen und zu überwinden «. Nach einem langen Zitat aus der »Abschweifung über produktive Arbeit《 stellt er erstaunt und fast ungläubig fest: "Doch heute gilt die Kriminalität als dem Sozialismus >wesensfremd. « ${ }^{8}$

Trotz der Rehabilitation von Marx durch die amerikanische Soziologie ruft die deutsche Rezeption immer noch politische Repressionen hervor. Fritz Sack, dem inzwischen Kategorien wie Klassenrecht« und Klassenjustiz « genauso leicht von den Lippen gehen wie »moderne Industriegesellschaft«, ist nach eige-

${ }^{3}$ Vol. 69 (1962), S. 172-181; wieder abgedrudt in: Sack/König (Hrsg.) -Kriminalsoziologie*, Ffm 1968 .

4 . Der Kriminelle ... ruft ein Solidaritätsgefühl unter denjenigen hervor, deren Einstellungen sonst auf divergierende Interessen gerichtet sind $\mathrm{Mead}$, zitiert nach Coser, a. a. O. S. 22.

3 .Critical Notes on the Cost of Crime «, in: Journal of Criminal Law and Criminology * 1936, S. $679-94$.

B E. M. Schur, »Reactions to Deviance: A Critical Assessment $<$ in: *The American Journal of Sociology *, Vol. 75 , 1970, S. 319.

7 Ffm 1968.

8 Günther Kaiser in einer Buchbesprechung in der KZfSS, 1968, S. 368. 
nen Angaben bereits seit dem "Symposium des Arbeitskreises Junger Kriminologen« I97I, auf dem er seinen »marxistisch-interaktionistischen Ansatz " vortrug, kaum noch wissenschaftlicher sondern vorrang politischer Kritik (Vorwurf des Extremismus, wobei unklar ist, ob Links- oder Rechtsextremismus gemeint ist) seitens der Fachkollegen ausgesetzt. ${ }^{9}$ Dabei ist Sack gewiß kein Marx-Epigone, sondern er entwickelt die marx'sche Position - schöpferisch? - weiter. Wie weiland Marx den Hegel, so hebt auch Sack die marx'sche Position dialektisch auf, stellt sie wieder auf den Kopf. Nicht der Verbrecher produziert den Richter, den Schergen, sondern nach Sack produziert der Scherge, der Richter, den Verbrecher: "Was sich in den Kriminalstatistiken ... niederschlägt, das sind ja die Ergebnisse von Verfahren, ... die die Eigenschaft (Kriminalität, Delinquenz), über die das spätere Urteil eine deskriptive Aussage macht, tatsächlich erst begründen und ins Leben rufen ". ${ }^{10}$

Trotz aller Anfeindungen halten junge Kriminologen weiterhin an Marx fest. In seiner Arbeit »Kriminalität ist normal« findet Haferkamp 1972 den Mut, die "Entökonomisierung der Soziologie und speziell der Soziologie des abweichenden Verhaltens «11 zu bedauern und betont, daß die Situationsdefinitionen der Kontrollinstanzen unschwer »mit den Ansprüchen des Wirtschaftssystems moderner Gesellschaften ${ }^{12}$ zu vermitteln seien. Offensichtlich eingeschüchtert, leistet er diese Vermittlung jedoch nicht mehr.

Dennoch: Im »Kriminologischen Journal« Heft $3 / 72$ geht man sogar soweit, einen Aufsatz von Hess "Die Lazarusschicht - Vorbemerkungen zum Problem der Armut « ausdrücklich mit der Begründung abzudrucken: »..., weil er das Problem der ,Armut unter Bedingungen kapitalistischer Arbeits- und Verwertungsprozesse stellt $\ll .{ }^{13}$

Sicher, diese Marx-Rezeption ist nicht aufzuhalten. Nur ist es der Satiriker Marx, der hier rezipiert wurde, nicht der Wissenschaftler. Daß die bürgerliche Wissenschaft sich in einer glossierenden Abschweifung wiedererkennt und damit ein Kapitel schwarzen Humors in den wissenschaftlichen Himmel hebt, ist nun selbst ein hübsches Beispiel unfreiwilliger Ironie.

Falco Werkentin

\section{Spendenaufruf des Initiativkomitees "Solidarität für Günter Amendt«}

Die Auftragsdruckerei des Springerkonzerns will später Rache nehmen. Sie hat ein rechtskräftiges BGH-Urteil gegen Günter Amendt erwirkt, das ihn als einzelnen aus der Masse einer Demonstration herausgreift und für ihn zur Folge hat, daß er DM 72000 zahlen soll. Das soll ihn in seiner bürgerlichen Existenz vernichten (indirektes Berufsverbot). In Wirklichkeit waren die Aktionen Ostern r 968 gegen die Auslieferung der Springerzeitungen eine kollektive und spontane Antwort auf die monatelange Hetze in den Springerblättern gegen die außer-

\footnotetext{
9 vgl. F. Sads $\times$ Definition von Kriminalität als politisches Handeln: der labeling approache, in: Kriminologisches Journal, Heft $1 / 1972$ bes. S. 4.

10 cbenda, S. 2 I.

11 Stuttgart 1972, S. 136.

12 ebenda, S. 107 .

13 S. I6I.
} 